\title{
Cantilever biosensor reader using a common-path, holographic optical interferometer
}

Pedersen, Henrik Chresten; Jakobsen, Michael Linde; Hanson, Steen Grüner; Dam-Hansen, Carsten; Olesen, Tom; Hansen, Poul

Published in:

Applied Physics Letters

Link to article, DOI:

$10.1063 / 1.3522888$

Publication date:

2010

Document Version

Publisher's PDF, also known as Version of record

Link back to DTU Orbit

Citation $(A P A)$ :

Pedersen, H. C., Jakobsen, M. L., Hanson, S. G., Dam-Hansen, C., Olesen, T., \& Hansen, P. (2010). Cantilever biosensor reader using a common-path, holographic optical interferometer. Applied Physics Letters, 97(22), 221110. https://doi.org/10.1063/1.3522888

\section{General rights}

Copyright and moral rights for the publications made accessible in the public portal are retained by the authors and/or other copyright owners and it is a condition of accessing publications that users recognise and abide by the legal requirements associated with these rights.

- Users may download and print one copy of any publication from the public portal for the purpose of private study or research.

- You may not further distribute the material or use it for any profit-making activity or commercial gain

- You may freely distribute the URL identifying the publication in the public portal 


\title{
Cantilever biosensor reader using a common-path, holographic optical interferometer
}

\author{
Henrik C. Pedersen, ${ }^{1, a)}$ Michael L. Jakobsen, ${ }^{1}$ Steen G. Hanson, ${ }^{1}$ Carsten Dam-Hansen, ${ }^{1}$ \\ Tom Olesen, ${ }^{2}$ and Poul Hansen ${ }^{2}$ \\ ${ }^{1}$ DTU Fotonik, Technical University of Denmark, 4000 Roskilde, Denmark \\ ${ }^{2}$ Unisensor A/S, Gydevang 42, 3450 Allerod, Denmark
}

(Received 25 May 2010; accepted 4 November 2010; published online 3 December 2010)

\begin{abstract}
We demonstrate an optical reader principle aimed at monitoring biologically induced deflections of microcantilevers often used in biosensor systems. The principle is based on a so-called common-path optical interferometer in which the two interfering optical beams are copropagating, which makes the system less sensitive to external disturbances. A detection limit of around $1 \mathrm{~nm}$ 's deflection is demonstrated. () 2010 American Institute of Physics. [doi:10.1063/1.3522888]
\end{abstract}

Microcantilever-based biosensors are devices that are capable of detecting binding affinities between biomolecules, such as proteins and DNA. ${ }^{1,2}$ Typically, an array of cantilevers is coated individually on one side with dedicated capture molecules that are able to bind to specific target biomolecules. Hence, upon exposure to a test specimen containing unknown agents, each cantilever will react differently, depending on the composition of target molecules in the sample. Upon attachment of target molecules, the cantilevers deflect due to surface tensions induced by the binding events. Typically, the observed deflections amount to some tens or hundreds of nanometers and a very sensitive reading device with a limit of detection around $1 \mathrm{~nm}$ is therefore needed to monitor the binding processes at sufficient accuracy. ${ }^{3-6}$

The most commonly used reader is the optical lever method, inspired by the read-out principle in atomic force microscopy. ${ }^{2,7}$ Here, the apex of each cantilever is illuminated by a laser beam that is reflected toward a position sensitive detector (PSD). Thus, a deflection of the cantilever results in a movement of the laser spot on the PSD. The optical lever method is extremely sensitive (limit-ofdetection down to $0.1 \mathrm{~nm}$ ). However, so far, the realization of high-density arrays has been hampered by the fact that each laser beam needs careful alignment with its corresponding PSD. One way to get around this problem was presented by Yue et $a l^{3}{ }^{3}$ who used one single laser beam to read out a two-dimensional array of ten cantilevers in parallel. The reflected ten beamlets were in this case simply captured by a charge-coupled device (CCD) camera and, hence, by using simple computer tracking algorithms, it was possible to monitor all ten static deflections at the same time. There are, however, two drawbacks using this technique. One is that the low bandwidth of the CCD camera (typically a few hertz) eliminates the possibility of dynamic read-out, in which case the cantilevers are set to vibrate in the kilohertz regime. ${ }^{2}$ Another drawback is that the achievable density of cantilevers is limited by diffraction of the beamlets. That is, the smaller the cantilevers-and thereby the beamlets-the larger the spots at the camera, which eventually will overlap and cause cross-talk.

A second technique is based on an array of lasers that are focused at a corresponding array of cantilevers from which

${ }^{\text {a)} E l e c t r o n i c ~ m a i l: ~ h c p e @ f o t o n i k . d t u . d k . ~}$ the beams are reflected toward a single PSD. The lasers are then time-multiplexed to make a sequential reading of the deflections. ${ }^{8}$ However, because the response of the PSD is very sensitive to initial positioning of the individual beams, this method is not suitable for large-numbered arrays, as each cantilever would require its own alignment.

In the present paper we demonstrate an alternative optical detection mechanism based on a so-called common-path interferometer that (i) does not require laborious alignment, (ii) potentially can be used in both dynamic and static modes, and (iii) can be applied in high-density arrays with very narrow cantilevers.

The basic reader setup is illustrated in Fig. 1. A vertical cavity surface emitting laser (VCSEL) (Optowell SS674U001, single mode, $\lambda=670 \mathrm{~nm}$, Jeonju-si, Korea) emits a diverging beam toward a collimating lens. After passing through a beam splitter the beam impinges on a holographic

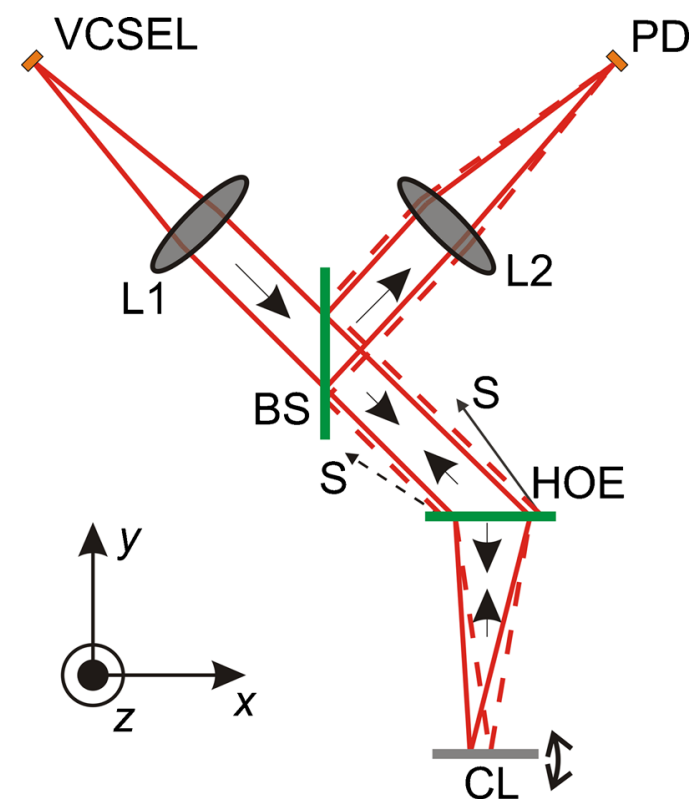

FIG. 1. (Color online) Basic principle of the cantilever reader, based on a holographic common-path interferometer. VCSEL = vertical cavity surface emitting laser, $\mathrm{L} 1=\mathrm{L} 2=$ lens (focal length $=25 \mathrm{~mm}$ ), BS=beam splitter, $\mathrm{HOE}=$ holographic optical element (double focusing element with focal length $=35 \mathrm{~mm}), \mathrm{CL}=$ cantilever, and $\mathrm{PD}=$ photo detector. The $\mathrm{S}$ rays represent secondary beams that are not used for detection. 


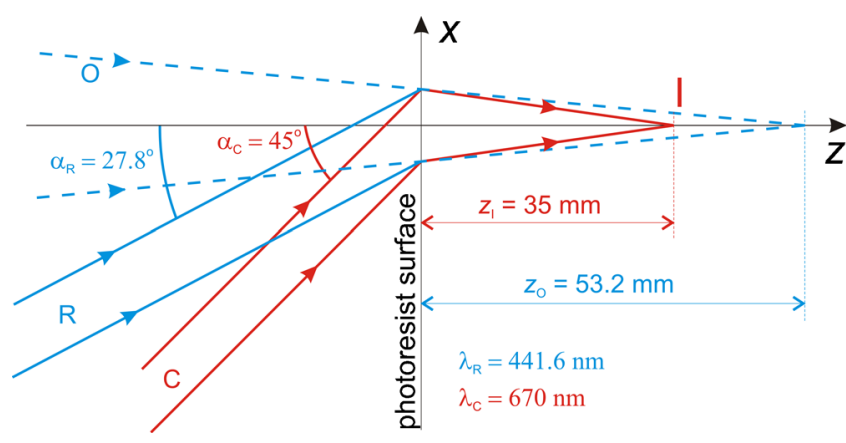

FIG. 2. (Color online) Recording beams $\mathrm{O}$ and $\mathrm{R}$ used for the HOE based on the desired reconstruction beams $\mathrm{C}$ and $\mathrm{I}$.

optical element (HOE) that comprises two superimposed holograms. The holograms are designed to generate two focused spots at the cantilever positioned $35 \mathrm{~mm}$ from the HOE. The spots are separated by $180 \mu \mathrm{m}$ along the cantilever.

The cantilever is metal-coated and, hence, reflects the two beams back toward the HOE as diverging beams. Here, the HOE converts each of the diverging beams into two collimated beams, of which two are codirectional. These beams are redirected by the beam splitter and focused by a second lens at a photodetector. As the beams originate from different spots on the cantilever, a deflection of the cantilever will introduce path length differences between the beams, causing the detected intensity to vary due to interference. This is the basic sensor principle. The off-directional secondary beams $\mathrm{S}$ are not used in this setup.

It should be noted that for clarity, the beam separations shown in Fig. 1 are highly exaggerated. In reality, the separations between the foci are $\sim 1 / 100$ of the HOE's focal length. As opposed to a conventional Michelson interferometer, this interferometer is referred to as a common-path type, ${ }^{9}$ since the out- and ingoing interfering beams are here propagating merely along the same paths. This is highly advantageous because thermally induced mechanical distortions in the setup as well as fluctuating air currents will tend to have identical influences on both beam paths, making the system less sensitive to these disturbances. Hence, drift problems are expected to be minimized.

The HOE is manufactured by using the design procedure for analog holography described by Latta. ${ }^{10}$ In our case we expose a photoresist film (Shipley SP1818, Towne Technologies, Sommerville, NJ) to two interfering laser beams from a $\mathrm{HeCd}$ laser (wavelength $=442 \mathrm{~nm}$ ), as illustrated in Fig. 2. Here, a collimated beam $\mathrm{R}$ with an angle of incidence of $27.8^{\circ}$ interferes with a focused beam $\mathrm{O}$ with a mean angle of incidence of $0^{\circ}$. The focal distance of the latter is $53.2 \mathrm{~mm}$ behind the film. This creates a hologram that focuses the collimated $670 \mathrm{~nm}$ beam at the desired $35 \mathrm{~mm}$ distance, as illustrated. After first exposure, the film is translated by $180 \mu \mathrm{m}$ along the $x$ direction, followed by a second exposure. In this manner, two identical holograms are superimposed in the film, resulting in two separated focused beams when the film is illuminated by the collimated VCSEL beam. Each spot has a diameter of $7 \mu \mathrm{m}\left(1 / e^{2}\right)$, as documented in Fig. 3 .

The cantilever used here was fabricated in SU-8 epoxy resin (MicroChem Inc., Newton, MA 02164-1418, USA) with a gold coating on top and with the dimensions $1 \times 50$

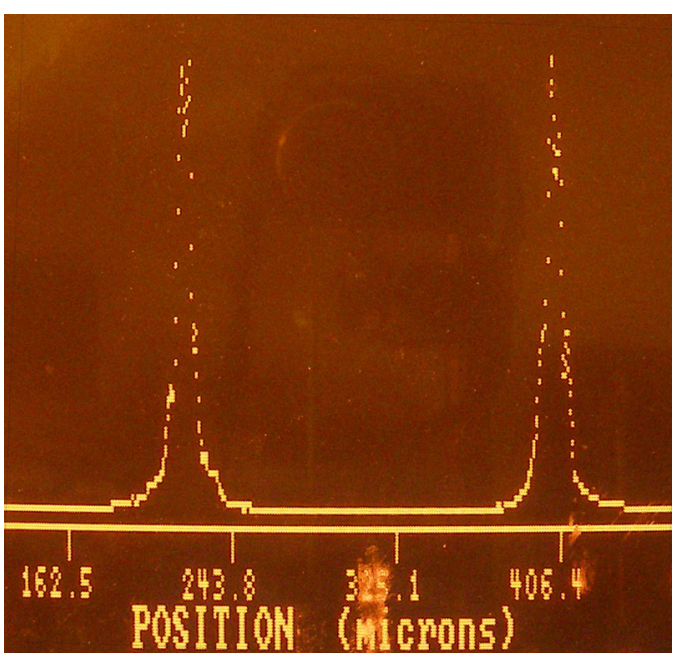

FIG. 3. (Color online) Intensity profiles of the two focused beams generated by the holographic optical element.

$\times 300 \mu \mathrm{m}$. Typically, it is desirable for biosensor applications to have a resolution down to $\sim 1 \mathrm{~nm}$ 's deflection of the cantilever. In order to test this, a cantilever chip is glued onto a $170 \mu \mathrm{m}$ thick microscope cover glass, as illustrated in Fig. 4(a). The tip of the cantilever is supported by a small SU-8 pedestal, in order to make a controlled lift of the tip. The glass plate is clamped to a tool that also holds a piezotranslator, at which the "free" end of the glass plate is resting. When the piezotranslator is moved up and down, a signifi-

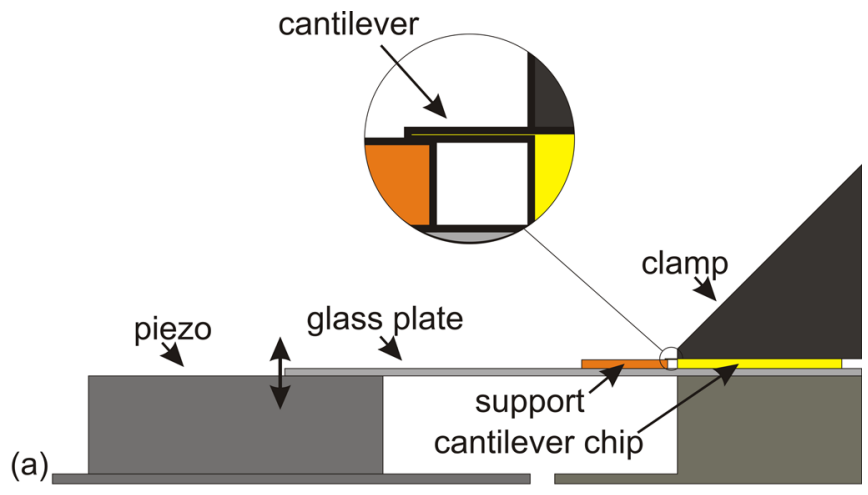

(b)

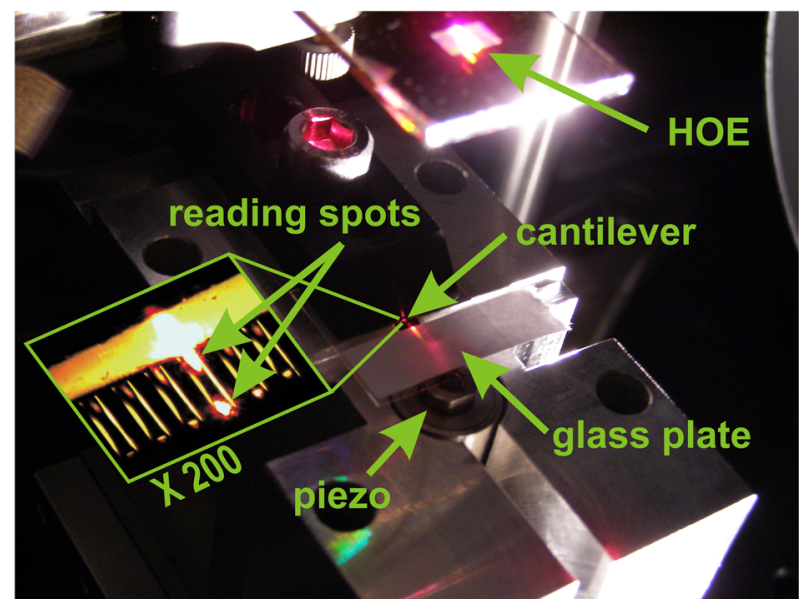

FIG. 4. (Color online) (a). Schematic of a piezo-based test rig for generating a controlled deflection of a cantilever at nanoscale. (b). Photograph of realized test rig; the inset shows a magnified picture of the two laser spots illuminating one of the cantilevers. 


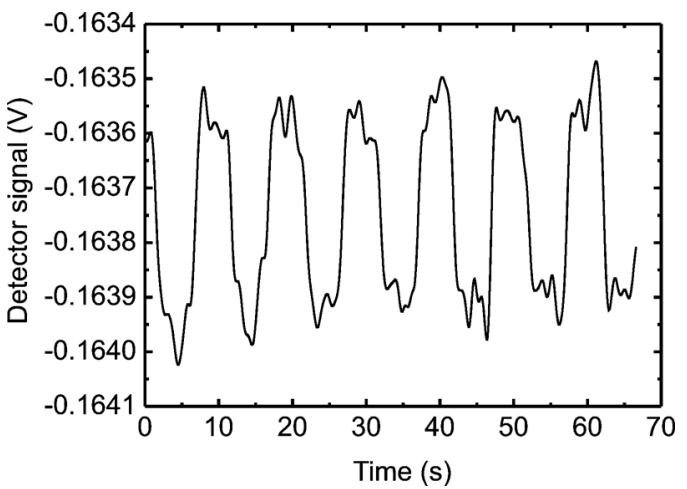

FIG. 5. Detector signal vs time as a result of a periodic deflection of the cantilever by $\pm 2 \mathrm{~nm}$.

cant down-scaling of the cantilever movement compared to the piezomovement is achieved because of the different distances to the pivot point of the glass plate. A photograph of the arrangement is shown in Fig. 4(b).

To calibrate the cantilever movement, a gradual lift is conducted that leads to a complete minimum-to-maximum sinusoidal variation of the detector signal. This corresponds to a total deflection of $\lambda / 4=167.5 \mathrm{~nm}$. The piezotranslator is subsequently set to give a $\pm 2 \mathrm{~nm}$ periodic cantilever deflection. The resulting detector signal is shown in Fig. 5. Here, it is clearly verified that deflections down to $1 \mathrm{~nm}$ may be resolved easily.

The reader system described above is easily extended to monitor arrays of cantilevers. This is achieved simply by replacing the single VCSEL by an array of VCSELs aligned along the $z$ axis, as shown in Fig. 6. Since the lens L1 and the HOE together work as a double-imaging system, the array of VCSELs is nicely double-imaged at the array of cantilevers. In a similar manner, the HOE together with the beam splitter

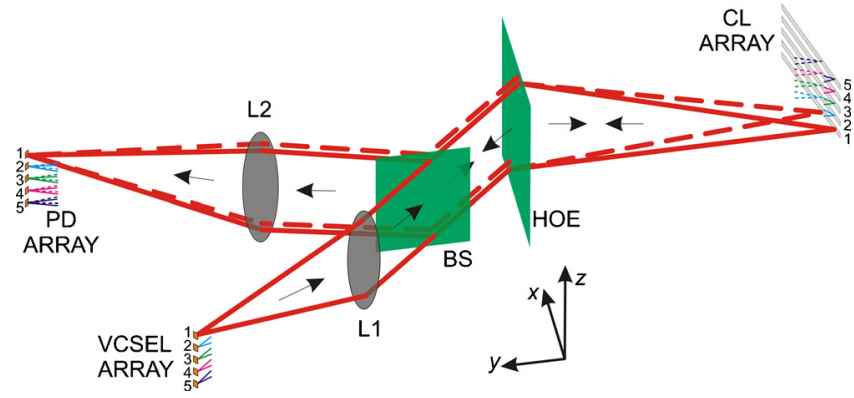

FIG. 6. (Color online) Schematic illustration of a holographic array reader. Here the transmitter system L1-HOE double-images an array of five VCSELs at an array of five cantilevers (for simplicity, only reading beams 1 are shown in full) after which the receiver system HOE-BS-L2 combines the reflected beams from the cantilevers at a corresponding photodetector array. The imaging properties of the two systems inhibit cross-talk. and the lens L2 act as a double-imaging system that combines the $2 \times 5$ array of reflected beams at the photodetector array. Note that the secondary beams, referred to as $\mathrm{S}$ beams in Fig. 1, are omitted in Fig. 6 for simplicity.

Because of the imaging properties, the system does not require very careful alignment. For example, an initial tilt of a cantilever does not displace the corresponding light spot at the detector, as is the case for the typical PSD-based systems. Moreover, as the detector array comprises normal photodiodes, the bandwidth is sufficiently high to be operated in dynamic reading mode, unlike a camera-based system. Finally, as all the spots on the cantilevers are imaged at their corresponding detectors, cross-talk is largely inhibited. This is very important for large-density cantilever arrays where cantilever spacings down to tens of microns are desirable.

The commercial potential of the system we believe is quite strong, mainly due to the system's large tolerance to varying initial deflections of the individual cantilevers. Moreover, the system is perfectly capable of working in both air and (transparent) liquids, just as in a conventional optical lever detection system.

In conclusion, we have reported a new cantilever reader system based on a common-path, holographic interferometer. It is demonstrated that the system is capable of detecting small deflections down to $1 \mathrm{~nm}$ and we have suggested a suitable extension of the system to encompass reading of cantilever arrays. The system can be used both in dynamic and static mode and it offers advantages compared to conventional systems in terms of reduced cross-talk.

The authors would like to thank A. Greve, NanoTech, Danish Technical University for providing the cantilever array as well as J. Stubager and P. Jensen, Fotonik, Danish Technical University for technical support. The work was supported by the Danish Council for Technology and Innovation under the Innovation Consortium CINO (Centre for Industrial Nano Optics) (Grant No. 75823).

${ }^{1}$ S. Xu and R. Mutharasan, Expert Opin. Drug Discov. 4, 1237 (2009).

${ }^{2}$ N. V. Lavrik, M. J. Sepanik, and P. G. Datskos, Rev. Sci. Instrum. 75, 2229 (2004)

${ }^{3}$ M. Yue, H. Lin, D. E. Dedrick, S. Satyanarayana, A. Majumdar, A. S. Bedekar, J. W. Jenkins, and S. Sundaram, J. Microelectromech. Syst. 13, 290 (2004).

${ }^{4}$ S. Lim, D. Raorane, S. Satyanarayana, and A. Majumdar, Sens. Actuators B 119, 466 (2006)

${ }^{5}$ J. Fritz, Analyst (Cambridge, U.K.) 133, 855 (2008).

${ }^{6}$ S. Koev, W. Bentley, and R. Ghodssi, Sens. Actuators B 146, 245 (2010). ${ }^{7}$ G. Meyer and N. M. Am, Appl. Phys. Lett. 53, 1045 (1988).

${ }^{8}$ M. K. Baller, H. P. Lang, J. Fritz, J. K. Gimzewski, U. Drechsler, H. Rothuizen, M. Despont, P. Vettiger, F. M. Battiston, J. P. Ramseyer, P. Fornaro, E. Meyer, and H.-J. Güntherodt, Ultramicroscopy 82, 1 (2000).

${ }^{9}$ S. R. Kitchen and C. Dam-Hansen, Proc. SPIE 4827, 112 (2002).

${ }^{10}$ J. N. Latta, Appl. Opt. 10, 599 (1971); 10, 609 (1971). 